\title{
The Ratio of C-Reactive Protein/Albumin is a Novel Inflammatory Predictor of Overall Survival in Cisplatin-Based Treated Patients with Metastatic Nasopharyngeal Carcinoma
}

\author{
Peng Sun, ${ }^{1,2}$ Cui Chen, ${ }^{3}$ Yi Xia, ${ }^{1,2}$ Xiwen Bi, ${ }^{1,2}$ Panpan Liu, ${ }^{1,2}$ Fei Zhang, ${ }^{1,2}$ Hang Yang, ${ }^{1,2}$ \\ Xin An, ${ }^{1,2}$ Wenqi Jiang, ${ }^{1,2}$ and Fenghua Wang ${ }^{1,2}$ \\ ${ }^{1}$ State Key Laboratory of Oncology in South China, Collaborative Innovation Center for Cancer Medicine, 651 Dong Feng RD East, \\ Guangzhou, Guangdong Province 510060, China \\ ${ }^{2}$ Department of Medical Oncology, Sun Yat-Sen University Cancer Center, 651 Dong Feng RD East, Guangzhou, \\ Guangdong Province 510060, China \\ ${ }^{3}$ Department of Oncology, The First Affiliated Hospital of Sun Yat-Sen University, 58 Zhongshan Road II, Guangzhou, \\ Guangdong Province 510080, China
}

Correspondence should be addressed to Wenqi Jiang; wenqi_jiang@163.com and Fenghua Wang; wangfh@sysucc.org.cn

Received 25 October 2016; Revised 5 February 2017; Accepted 26 February 2017; Published 6 June 2017

Academic Editor: Szilárd Nemes

Copyright @ 2017 Peng Sun et al. This is an open access article distributed under the Creative Commons Attribution License, which permits unrestricted use, distribution, and reproduction in any medium, provided the original work is properly cited.

\begin{abstract}
The C-reactive protein/albumin (CRP/Alb) ratio has been recently identified as a prognostic factor in various cancers, whereas its role remains unclear in metastatic nasopharyngeal carcinoma (NPC). The current study retrospectively analyzed 148 patients with metastatic NPC who underwent cisplatin-based chemotherapy and further evaluated the prognostic value of the CRP/Alb ratio and its association with clinical characteristics in these patients. The optimal cut-off value was 0.189 for the CRP/Alb ratio. The high CRP/Alb ratio was significantly associated with elevated NLR, platelet-to-lymphocyte ratio (PLR), and EBV-DNA levels and decreased haemoglobin level (all $p<0.05$ ). The results of multivariate analysis showed that the CRP/Alb ratio was an independent prognostic factor of overall survival. Patients with a high CRP/Alb ratio $(\geq 0.189)$ had a 1.867 times $(p=0.024,95 \%$ $\mathrm{CI}=1.085-3.210)$ greater risk of mortality compared with those with a low CRP/Alb ratio $(<0.189)$. In addition, combining the $\mathrm{CRP} / \mathrm{Alb}$ ratio with GPS could accurately discriminate the prognosis of our patients. Our results suggested that the CRP/Alb ratio is a feasible and inexpensive tool for predicting survival outcomes and is a valuable coadjutant for the GPS to further identify differences in survivals of patients with metastatic NPC.
\end{abstract}

\section{Introduction}

Nasopharyngeal carcinoma (NPC) is a distinct head and neck cancer with unique etiological and epidemiological feathers, with the incidence varying from $0.5-3 / 100,000$ per year in North Africa to 20-30/100,000 in certain epidemic areas, such as South China and Southeast Asia [1-4]. Radiotherapy is the primary treatment of NPC, and the development of intensity-modulated radiotherapy technology (IMRT) and new chemotherapy agents significantly improves the cure rate [5-7]. However, more than $20 \%$ of NPC patients will ultimately develop distant metastasis after definitive chemoradiotherapy [8]. On the other hand, $5 \%-6 \%$ of NPC patients have disseminated disease at the time of initial diagnosis [8]. Hence, metastatic disease remains a critical problem and a leading cause of death among patients with NPC. For patients with metastatic NPC, systematic chemotherapy is the standard therapeutic option and has been well established [9]. Although multiple agents and regimens have been explored, the palliative chemotherapy for NPC has rarely been studied in any randomized trials yet. Cisplatinbased multiple chemotherapy was reported to reach a response rate of $50-80 \%$ in NPC patients; it has been recommended as the standard first-line regimen for metastatic NPC [10-12]. However, the clinical outcome varied 
significantly in patients with metastatic NPC. Therefore, it is critically important to find predictive factors for prognosis, which could help us to accurately select the patients who respond well to cisplatin-based chemotherapy.

Since the well-known tumor-node-metastasis (TNM) staging system could not provide prognostic information, a series of clinical characteristics and laboratory biomarkers associated with prognosis of patients with metastatic NPC have been explored in several studies [13-19]. Hemoglobin, performance status (PS), and disease-free interval (DFI) were first identified as prognostic factors for disseminated NPC by Toh et al. [20] in 2005. Then, lactate dehydrogenase (LDH) was also found to be significantly associated with survival of patients with metastatic NPC [21, 22]. Epstein-Barr virus (EBV) was a steady prognostic factor and predictor of treatment response for patients with metastatic NPC who underwent palliative chemotherapy [21, 23, 24]. In 2012, Jin et al. [21] built a prognostic model including EBVDNA load, PS, LDH, alkaline phosphatase (ALP), and hemoglobin and successfully stratified the patients into different risk groups.

The role of systematic inflammatory response has been increasingly appreciated in multiple cancers; inflammatory biomarkers, such as C-reactive protein (CRP) level [25], Glasgow Prognostic Score (GPS) [26], modified Glasgow Prognostic Score (mGPS) [27], neutropil to lymphocyte ratio (NLR) [26], and monocyte to lymphocyte ratio (MLR) [28, 29], have already showed prognostic value in metastatic NPC. Xia et al. [25] retrospectively analyzed 116 patients with metastatic NPC who underwent palliative chemotherapy and found that baseline CRP level was significantly associated with survival. In 2014, Chen et al. [26] reviewed 211 Chinese patients with disseminated NPC who all received cisplatin-based chemotherapy and further validated the prognostic value of GPS and NLR. Recently, the CRP/albumin (CRP/Alb) ratio was recognized as a novel inflammatory index in infectious disease $[30,31]$. In addition, the CRP/Alb ratio has showed promising prognostic value in gastric cancer [32], esophageal cancer [33], colorectal cancer [34], small cell lung cancer [35], and even in nonmetastatic NPC [36].

However, to the best of our knowledge, there is no data regarding the prognostic value of the CRP/Alb ratio in the metastatic NPC. Therefore, we conducted this study and aimed to examine the prognostic value of the CRP/Alb ratio and its association with clinical characteristics in patients with disseminated NPC.

\section{Patients and Methods}

2.1. Ethics Approval and Consent to Participate. All the patients offered informed consent for collection of clinical characteristics and pathologic information. The study was approved by the Bioethics Committee of the Sun Yat-Sen University Cancer Center for a retrospective analysis of the collected data. The study was undertaken in accordance with the ethical standards of the World Medical Association's Declaration of Helsinki.
2.2. Patients. We retrospectively analyzed the records of patients with NPC who were treated at the Sun Yat-Sen University Cancer Center between January 2008 and October 2011. All the patients met the following criteria: (a) the disease was pathologically diagnosed as NPC and evaluated clinically to be metastatic disease; (b) complete pretreatment data of CRP and albumin were available; (c) at least two cycles of first-line cisplatin-based palliative chemotherapy were administrated; (d) Karnofsky Performance Scores (KPS) were $\geq 60$; and (e) hepatic and renal functions were normal. Exclusion criteria were as follows: (a) patients with brain metastases; (b) patients with other types of malignancy; or (c) patients with clinical evidence of infection or other inflammatory disease. Finally, a total of 148 patients were identified and originated the current study cohort.

2.3. Treatment and Follow-Up. The following three cisplatinbased chemotherapy regimens were administrated every 21 days:

(a) TP regimen: paclitaxel $\left(175 \mathrm{mg} / \mathrm{m}^{2}\right.$ intravenously (IV) over 3 hours with standard premedication on day 1 of a 21 -day cycle) plus cisplatin $\left(25 \mathrm{mg} / \mathrm{m}^{2} \mathrm{IV}\right.$ on days 1-3 of a 21-day cycle)

(b) PF regimen: cisplatin $\left(25 \mathrm{mg} / \mathrm{m}^{2} \mathrm{IV}\right.$ on days $1-3$ of a 21 -day cycle) plus 5 -fluorouracil $\left(500 \mathrm{mg} / \mathrm{m}^{2} \mathrm{IV}\right.$ on days $1-5$ of a 21 -day cycle)

(c) TPF regimen: paclitaxel $\left(135 \mathrm{mg} / \mathrm{m}^{2} \mathrm{IV}\right.$ over 3 hours with standard premedication on day 1 of a 21-day cycle) plus cisplatin $\left(25 \mathrm{mg} / \mathrm{m}^{2}\right.$ IV on days $1-3$ of a 21-day cycle) plus 5-fluorouracil $\left(800 \mathrm{mg} / \mathrm{m}^{2}\right.$, continuous IV infusion for 24 hours, on days 1-5 of a 21-day cycle).

After treatment, all patients were followed up every 3 months. Physical and radiological examinations were performed every 3 months or when clinical progression of disease was indicated. The last follow-up was performed on December 31, 2013. Overall survival (OS) was defined as the time interval from the initial diagnosis of metastasis to the date of death or the last follow-up.

2.4. Evaluation. Basic demographics (gender, age), baseline clinical characteristics (KPS, site of metastasis), and relevant laboratory data were collected. Hematological parameters, biochemical markers, and EBV-DNA load were examined within one week before chemotherapy. Smoking status was recorded as ever or never for all patients. Smoker ever was defined as $\geq 1$ lifetime pack-year. The GPS, NLR, and platelet-to-lymphocyte ratio (PLR) were calculated. We calculated the CRP/Alb ratio as dividing the CRP level $(\mathrm{mg} / \mathrm{L})$ by the serum albumin level $(\mathrm{g} / \mathrm{L})$. The Response Evaluation Criteria in Solid Tumors (RECISTs) 1.0 was used to evaluate tumor response.

2.5. Statistical Analyses. Continuous variables, such as $\mathrm{LDH}$ and NLR, are presented as median value and range and were compared by using $t$-test or nonparametric test. Categorical 
TABLE 1: Baseline clinical features of 148 patients with metastatic nasopharyngeal carcinoma.

\begin{tabular}{|c|c|c|c|c|}
\hline Characteristic & Number (\%) & CRP/Alb $<0.189$, number $(\%)$ & CRP/Alb $\geq 0.189$, number $(\%)$ & $p$ value \\
\hline Gender (male/female) & $124 / 24(83.8 / 16.2)$ & $63 / 15(80.8 / 19.2)$ & $61 / 9(87.1 / 12.9)$ & 0.373 \\
\hline Age, years (median/range) & $45 / 24-72$ & $45 / 26-70$ & $43.5 / 24-72$ & 0.765 \\
\hline KPS (median/range) & $90 / 60-100$ & $90 / 60-100$ & $90 / 60-100$ & 0.276 \\
\hline Number of involved sites (one/multiple) & $69 / 79(46.6 / 53.4)$ & $42 / 36(53.8 / 46.2)$ & $27 / 43(38.6 / 61.4)$ & 0.071 \\
\hline Synchronous metastasis (yes/no) & $43 / 105(29.1 / 70.9)$ & $20 / 58(25.6 / 74.4)$ & $23 / 47(32.9 / 67.1)$ & 0.368 \\
\hline Liver metastasis (yes/no) & $56 / 92(37.8 / 62.2)$ & $28 / 50(35.9 / 64.1)$ & $28 / 42(40 / 60)$ & 0.615 \\
\hline Lung metastasis (yes/no) & $69 / 79(46.6 / 53.4)$ & $38 / 40(48.7 / 51.3)$ & $31 / 39(44.3 / 55.7)$ & 0.623 \\
\hline Bone metastasis (yes/no) & $61 / 87(41.2 / 58.8)$ & $28 / 50(35.9 / 64.1)$ & $33 / 37(47.1 / 52.9)$ & 0.184 \\
\hline Smoking (yes/no) & $68 / 80(45.9 / 54.1)$ & $36 / 42(42.1 / 53.8)$ & $32 / 38(45.7 / 54.3)$ & 1.000 \\
\hline GPS $(0 / 1 / 2)$ & $88 / 46 / 14(59.5 / 31.1 / 9.4)$ & $52 / 20 / 6(66.7 / 25.6 / 7.7)$ & $36 / 26 / 8(51.4 / 37.1 / 11.4)$ & 0.169 \\
\hline Serum LDH, U/L (median/range) & $202.5 / 25-2975$ & $179.5 / 122-2821$ & $230 / 25-2975$ & 0.069 \\
\hline EBV-DNA, copies/mL (median/range) & $4.82 \times 104 / 0-9.13 \times 107$ & $3.49 \times 104 / 0-9.87 \times 106$ & $5.32 \times 105 / 0-9.13 \times 107$ & $0.013^{*}$ \\
\hline Chemotherapy regimen (TP/PF/TPF) & $12 / 45 / 91(8.1 / 30.4 / 61.5)$ & $7 / 26 / 45(9 / 33.3 / 57.7)$ & $5 / 19 / 46(7.2 / 27.1 / 65.7)$ & 0.605 \\
\hline Treatment response $(\mathrm{CR}+\mathrm{PR} / \mathrm{PD}+\mathrm{SD})$ & $107 / 41(72.3 / 27.7)$ & $59 / 19(75.6 / 24.4)$ & $48 / 22(68.6 / 31.4)$ & 0.363 \\
\hline NLR (median/range) & $3.3 / 1-35.2$ & $2.8 / 1-14$ & $4 / 1-35.2$ & $0.001^{*}$ \\
\hline PLR (median/range) & $181 / 56.4-820$ & $161 / 88-735$ & $231 / 56.4-820$ & $0.001^{*}$ \\
\hline Hemoglobin, g/L (median/range) & $131 / 43-171$ & $132 / 43-171$ & $123 / 82-162$ & $0.019^{*}$ \\
\hline
\end{tabular}

CRP/Alb: C-reactive protein/albumin ratio; KPS: Karnofsky Performance Score; GPS: Glasgow Prognostic Score; NLR: neutrophil to lymphocyte ratio; PLR: platelet-to-lymphocyte ratio; ${ }^{*} p<0.05$.

variables, such as gender and GPS, were described as the numbers and percentages and were compared by using the chi-square or Fisher's exact test.

A web-based system, R software-engineered, designed by Budczies J et al. [37], was used to identify the optimal cutoff value of potential prognostic parameters (http://molpath. charite.de/cutoff/). The Kaplan-Meier method was used to estimate the OS. Univariate and multivariate survival analyses were performed based on the Cox proportional hazards regression methodology. Hazard ratios (HRs) with 95\% CIs and two-sided $p$ values were reported. An alpha value of $p<0.05$ was considered statistically significant. The statistical analyses were performed using the Statistical Package for the Social Sciences version 19.0 (IBM, Armonk, NY, USA).

\section{Results}

3.1. Patient Characteristics. A total of 148 patients with metastatic NPC were included in our study. Of these, 124 $(83.8 \%)$ were males and $24(16.2 \%)$ were females; 68 $(45.9 \%)$ were smokers and $80(54.1 \%)$ were nonsmokers. The median age at initial diagnosis of metastatic NPC was 45 years (ranging 24-72 years). More than half $(n=79$, $53.4 \%$ ) of the patients had more than one metastasis site, and few of the patients $(n=43,29.1 \%)$ developed synchronous metastasis. Liver metastasis, lung metastasis, and bone metastases were found in 56 patients (37.8\%), 69 patients (46.6\%), and 61 patients (41.2\%), respectively. The baseline plasma EBV-DNA load ranged from 0 to $9.13 \times 10^{7}$ copies $/ \mathrm{mL}$, with a median value of $4.82 \times 10^{4}$ copies/mL. GPS was evaluated as 0 in 88 patients (59.5\%), 1 in 46 patients (31.1\%), and 2 in 14 patients
(9.4\%). The median values of the CRP/Alb, NLR, and PLR were $0.164,3.3$, and 181 , respectively. Of the 148 patients, $12(8.1 \%)$ were treated with the TP regimen, 45 (30.4\%) with the PF regimen, and 91 (61.5\%) with the TPF regimen (Table 1 ).

3.2. Survival. Using the Cutoff Finder, we determined 0.189 as the optimal cutoff value of the CRP/Alb ratio for OS. All participants were then divided into the high CRP/Alb ratio group $(\geq 0.189, n=70)$ and low $\mathrm{CRP} / \mathrm{Alb}$ ratio group $(<0.189, n=78)$. Accordingly, binarization of other continuous data (LDH, NLR) by optimal cutoff value was performed for the subsequent analysis of OS (Table 2).

Median follow-up time was 15.3 months (ranging 1 month-66 months). Of the 148 patients, 77 (52.0\%) died before the last follow-up. Median OS time for the entire patient group were 21.8 months, with the 1 - and 2 -year OS rates of $69.6 \%$ and $22.3 \%$, respectively. Compared with the patients in the high $\mathrm{CRP} / \mathrm{Alb}$ ratio group, patients in the low CRP/Alb ratio group had significantly longer overall survival (25.2 months versus 19.5 months, $p=0.003$ ) (Figure 1).

Besides the CRP/Alb ratio, GPS $(p<0.001)$, serum LDH level $(p=0.006)$, plasma EBV-DNA load $(p<0.001)$, pretreatment NLR $(p=0.017)$, PLR $(p=0.002)$, and treatment response $(p=0.005)$ were associated with OS in the univariate analysis. After adjusting for other covariates in multivariate analysis, the $\mathrm{CRP} / \mathrm{Alb}$ ratio $(p=0.024)$ was proved to be an independent prognostic factor of OS, as well as GPS $(p=0.001)$ and EBV-DNA load $(p=0.041)$. We found that patients in the high CRP/Alb ratio group had a 1.867 times $(p=0.024,95 \% \mathrm{CI}=1.085-3.210)$ greater risk of mortality compared with those in the low CRP/Alb ratio group (Table 2). 
TABLE 2: Univariate and multivariate analysis of OS in 148 patients with metastatic nasopharyngeal carcinoma.

\begin{tabular}{|c|c|c|c|c|}
\hline \multirow{2}{*}{ Variable } & \multicolumn{2}{|c|}{ Univariate } & \multicolumn{2}{|c|}{ Multivariate } \\
\hline & $p$ value & HR $(95 \% \mathrm{CI})$ & $p$ value & HR $(95 \% \mathrm{CI})$ \\
\hline Gender (male/female) & 0.570 & $0.83(0.435-1.581)$ & & \\
\hline Age, years $(<50 / \geq 50)$ & 0.773 & $1.078(0.646-1.801)$ & & \\
\hline KPS $(\geq 90 /<90)$ & 0.411 & $0.765(0.403-1.451)$ & & \\
\hline Number of involved sites (one/multiple) & 0.179 & $1.369(0.866-2.164)$ & & \\
\hline Synchronous metastasis (yes/no) & 0.168 & $0.704(0.427-1.160)$ & & \\
\hline Liver metastasis (yes/no) & 0.802 & $0.942(0.593-1.498)$ & & \\
\hline Lung metastasis (yes/no) & 0.389 & $1.219(0.777-1.914)$ & & \\
\hline Bone metastasis (yes/no) & 0.071 & $1.525(0.965-2.411)$ & & \\
\hline Smoking (yes/no) & 0.210 & $0.750(0.478-1.177)$ & & \\
\hline GPS $(0 / 1 / 2)$ & $<0.001^{*}$ & $3.135(2.309-4.256)$ & $0.001^{*}$ & $2.137(1.369-3.337)$ \\
\hline CRP/Alb $(<0.189 / \geq 0.189)$ & $0.003^{*}$ & $1.998(1.253-3.185)$ & $0.024^{*}$ & $1.867(1.085-3.210)$ \\
\hline Serum LDH, U/L $(<212 / \geq 212)$ & 0.006 & $1.880(1.193-2.962)$ & 0.602 & $0.858(0.483-1.524)$ \\
\hline EBV-DNA, copies/mL $(<4.82 \times 104 / \geq 4.82 \times 104)$ & $<0.001^{*}$ & $4.554(2.792-7.427)$ & $0.041^{*}$ & $2.012(1.027-3.941)$ \\
\hline Chemotherapy regimen (PF/TP/TPF) & 0.644 & $0.922(0.653-1.302)$ & & \\
\hline Treatment response $(\mathrm{CR}+\mathrm{PR} / \mathrm{PD}+\mathrm{SD})$ & $0.005^{*}$ & $1.984(1.231-3.197)$ & 0.420 & $1.242(0.734-2.101)$ \\
\hline $\operatorname{NLR}(<5 / \geq 5)$ & $0.017^{*}$ & $2.039(1.133-3.671)$ & 0.192 & $1.522(0.810-2.858)$ \\
\hline $\operatorname{PLR}(<152 / \geq 152)$ & $0.002^{*}$ & $2.450(1.407-4.267)$ & 0.128 & $1.617(0.870-3.003)$ \\
\hline Hemoglobin, $\mathrm{g} / \mathrm{L}(<11 / \geq 11)$ & 0.585 & $1.172(0.664-2.069)$ & & \\
\hline
\end{tabular}

CRP/Alb: C-reactive protein/albumin ratio; KPS: Karnofsky Performance Score; GPS: Glasgow Prognostic Score; NLR: neutrophil to lymphocyte ratio; PLR: platelet-to-lymphocyte ratio; LDH: lactate dehydrogenase; ${ }^{*} p<0.05$.

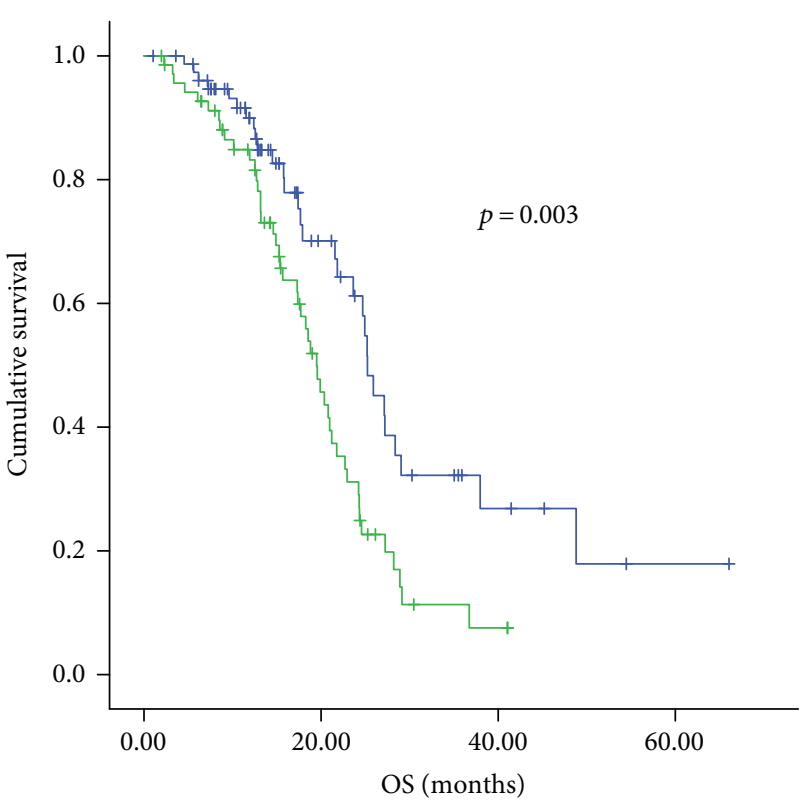

$\mathrm{CRP} / \mathrm{Alb}$

$$
\begin{aligned}
& \neg<0.189 \\
& -\neg \geq 0.189
\end{aligned}
$$

Figure 1: Kaplan-Meier curves for overall survival (OS) according to the CRP/Alb ratio.

3.3. Association of the CRP/Alb Ratio with Clinicopathologic Characteristics. Patient characteristics were compared between subgroups by the CRP/Alb ratio (Table 1). It was found that the high CRP/Alb ratio showed a trend to correlate with multiple metastasis $(p=0.071)$ and elevated LDH level $(p=0.069)$, with marginal statistical significance. Of note, the high CRP/Alb ratio was significantly associated with high NLR, PLR, and EBV-DNA level and low haemoglobin level (all $p<0.05$ ).

3.4. Generation of a New Prognostic Score. Although GPS was an independent prognostic factor in the Cox model, the difference in OS between patients with GPS 1 and those with GPS 2 was not statistically significant (15.8 months versus 8.5 months, $p=0.101$, Figure 2(a)). Thus, we further added the CRP/Alb ratio to the GPS system and established a new prognostic score called aGPS. A score of 1 was assigned to CRP $>10 \mathrm{mg} / \mathrm{L}$, albumin $<35 \mathrm{~g} / \mathrm{L}$, and the CRP/Alb ratio $\geq 0.189$. Thus, of the 148 patients, $52(35.1 \%)$ were evaluated as having an aGPS of $0,56(37.8 \%)$ had an aGPS of $1,32(21.6 \%)$ had an aGPS of 2, and $8(5.4 \%)$ had an aGPS of 3 ; the median OS of these patients were 28.4 months, 21.2 months, 14.9 months, and 6.1 months, respectively $(p<0.001$, Figure 2(b)). The OS curves of these subgroups were statistically separated from each other. Furthermore, aGPS (aGPS $=0$ versus aGPS $>0$ ) was also significantly associated with prognosis of NPC patients when stratified by plasma EBV-DNA level, whereas plasma EBV-DNA level $\left(<4.82 \times 10^{4}\right.$ copies $/ \mathrm{mL}$ versus $\geq 4.82 \times 10^{4}$ copies $\left./ \mathrm{mL}\right)$ was not (Figure 3). Patients with aGPS 0 had longer OS than patients with aGPS $>0$ either in the high plasma EBV-DNA level group or in the low plasma EBV-DNA level group. 


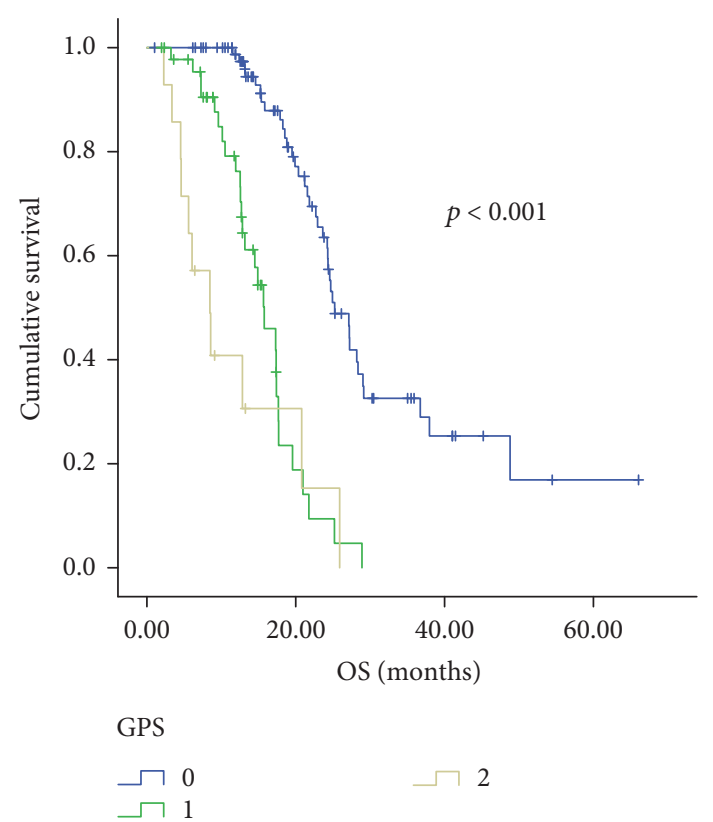

(a)

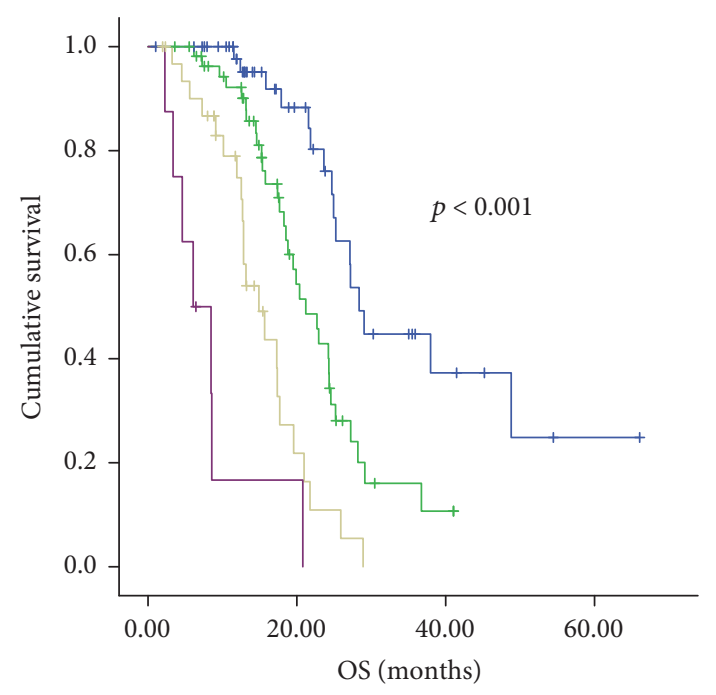

aGPS

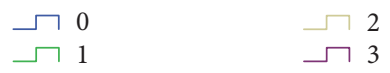

(b)

FIGURE 2: Kaplan-Meier curves for overall survival (OS) according to the GPS (a) and to the aGPS (b).
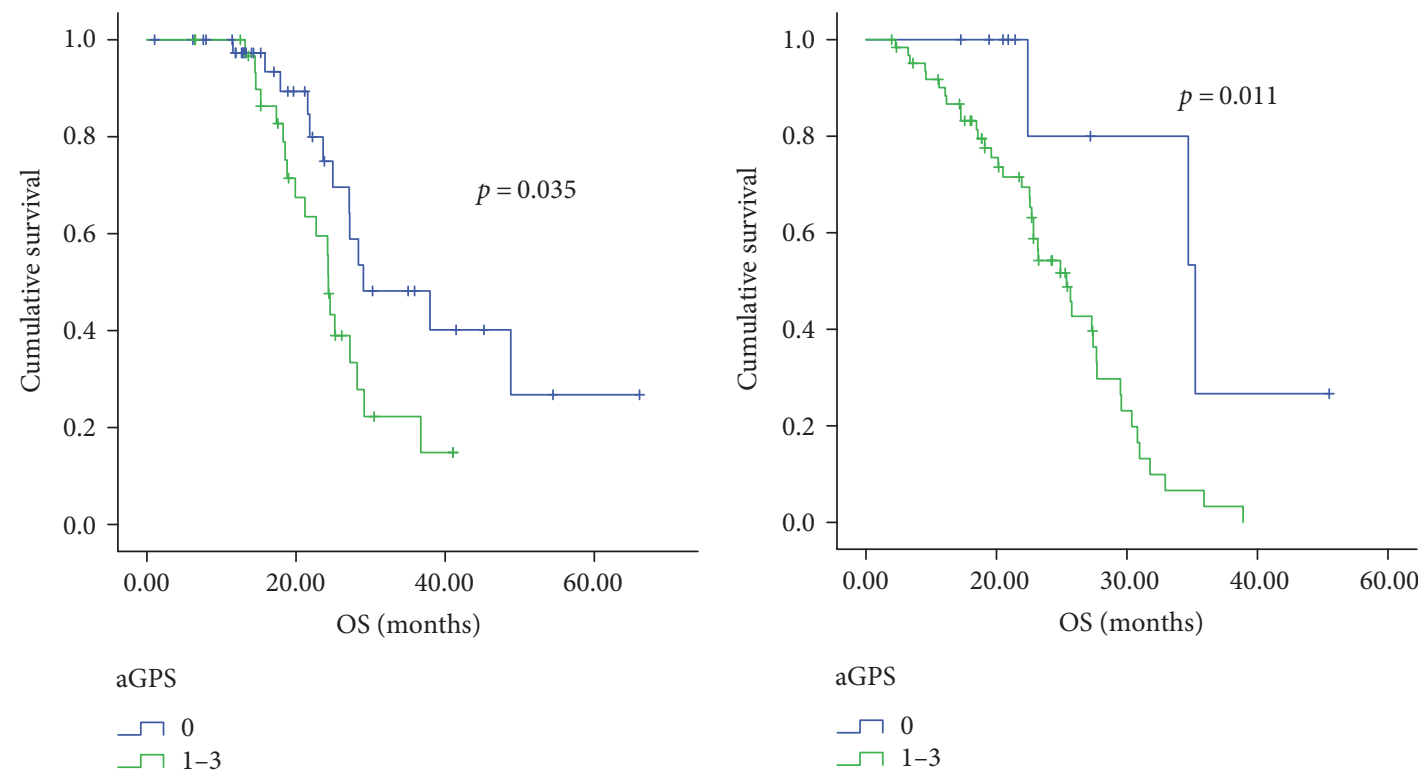

(a)

(b)

FIGURE 3: Kaplan-Meier curves for overall survival (OS) according to the aGPS in patients with pretreatment EBV DNA $<4.82 \times 10^{4}$ copies $/ \mathrm{mL}$ (a) and in those with pretreatment EBV DNA $\geq 4.82 \times 10^{4}$ copies $/ \mathrm{mL}$ (b).

\section{Discussion}

Systematic inflammatory response has been well studied in the carcinogenesis and progression of malignancies during the past decade, and various inflammatory biomarkers have shown to be associated with cancer prognosis [38]. Serum CRP is an acute-phase protein produced by hepatocytes; it has been long recognized as a prognostic factor for many patients with malignancies including NPC [25, 39-42]. Meanwhile, hypoalbuminemia could predominantly reflect malnutrition as well as inflammatory condition and was found to be associated with impaired survival outcome of cancer patients [43]. GPS and mGPS were thus generalized from the combination of CRP and albumin; as new inflammatory markers, they have been proved to be useful prognostic factors to predict the risk of cancer. 
More recently, a novel combination model of CRP and albumin-CRP/Alb ratio-has also been increasingly appreciated since it could serve as a predictor of a clinical outcome in patients with serious infectious disease and those with cancers [31, 32, 34, 35, 44]. He et al. [36] investigated the CRP/Alb ratio in nonmetastatic NPC. They found that the CRP/Alb ratio was a steady prognostic factor for the prognosis of patients with nonmetastatic NPC, and it could identify survival differences even if stratified by EBV-DNA level. However, most of previous studies on the CRP/Alb ratio limited the cohort to patients who were diagnosed with localized cancers and underwent curative therapy $[32,34,44]$. By contrast, our study focused on the patients with metastatic NPC and firstly demonstrated that the $\mathrm{CRP} / \mathrm{Alb}$ ratio was an independent prognostic factor of overall survival.

Of note, the CRP/Alb ratio reflects a comprehensive state of both CRP and albumin rather than a simple addition of CRP and albumin. In contrast to GPS, the CRP/Alb ratio might indicate more comprehensive information, reflect dynamic change of systematic inflammation, and identify tiny difference among a large patient population. Although GPS was also found to be independently associated with OS $(p=0.001)$ as well as the CRP/Alb ratio, there were $59.3 \%$ of patients classified in the group of GPS 0 , which implied that the GPS could not accurately predict the prognosis of a large proportion of our patients. Moreover, the GPS was not statistically different in the OS between the patients with GPS 1 and those with GPS 2. More interestingly, we could enrich the prognostic information of CRP-albumin-based model (aGPS) when we introduced the CRP/Alb ratio into the model along with GPS. Similar findings were also reported by Wei et al. [45], in which the patients with GPS 0 could be categorized into two risk subgroups by the CRP/ Alb ratio. Furthermore, aGPS was significantly associated with OS when stratified by plasma EBV-DNA level in our cohort. From this point of view, aGPS which was consisted of three parameters (CRP, albumin, and CRP/Alb ratio) might serve as a more accurate prognostic factor than GPS and provide new insight into the inflammatory prognostic system. However, the prognostic efficacy of aGPS should be further explored in prospective studies and in other cancers other than in NPC.

The association of the CRP/Alb ratio with other clinical characteristics was also analyzed in our study. It was found that the high CRP/Alb ratio subgroup had significantly higher NLR, PLR, and pretreatment EBV-DNA level and lower hemoglobin level compared with the low CRP/Alb ratio subgroup. Based on these findings, it was suggested that the CRP/Alb ratio could not only reflect the individual's systematic inflammatory response but also perform as an indicator of nutritional status.

However, inconsistent with the results of $\mathrm{Xu}$ et al. [33] and Wei et al. [45] studies, our data did not show a significant association between GPS and the CRP/Alb ratio. From this point, it was suggested that the combination of GPS and the CRP/Alb ratio might be more informative on inflammation and nutrition than either one alone in NPC patients.
In contrast to localized NPC with curative treatment, the prognostic factors were less identified in metastatic NPC. Plasma EBV-DNA load has been long established as a strong indicator of tumor burden and as a reliable prognostic factor in both disseminated NPC and localized NPC [4, 21, 24]. In line with these data, high pretreatment EBV-DNA level was also found to be an unfavourable prognostic factor in our study. Besides EBV-DNA level, other prognostic factors such as LDH level [21, 22] and NLR [26] have been identified in metastatic NPC. Baseline LDH level, haemoglobin level, number of metastasis site, NLR, and PLR were also analyzed in the current study and showed a close association with the $\mathrm{CRP} / \mathrm{Alb}$ ratio. However, they failed to independently predict prognosis in the multivariate analysis, indicating that they reflect tumor burden less sensitively and less precisely than the $\mathrm{CRP} / \mathrm{Alb}$ ratio.

The optimal cutoff value for the CRP/Alb ratio varied in different studies. Even in the two studies of esophageal squamous cell carcinoma (ESCC), the cutoff values were different. In the study by $\mathrm{Xu}$ et al. [33], the cutoff was set as 0.50 , which was much higher than that in the study by Wei et al. (0.095) [45], due to different methods and different patient population. In our study, we used the same method as Wei et al. [45] and determined the cutoff value as 0.189 . In the study investigating the CRP/Alb ratio in nonmetastatic NPC, 0.064 was determined as the optimal cutoff value [36].

Generally speaking, there were three main superiorities in this study. First, we explored the association of the $\mathrm{CRP} / \mathrm{Alb}$ ratio with treatment response and chemotherapy regimen, which was not always discussed in previous studies. Second, all patients underwent at least two cycles of cisplatin-based chemotherapy, and the heterogeneity of therapeutic options might minimize the potential interference from the variation of different treatments. By contrast, most of previous studies included patients with noncisplatin chemotherapy. Xia et al. [25] enrolled two patients who underwent sorafenib, which was not recommended as standard first-line treatment. In the study by Jin et al. [21], 5\% of the patients received monotherapy with capecitabine for their poor performance status. Toh et al. [20] did not describe the detailed treatment for their study cohorts, and $40 \%$ of the patients did not undergo any salvage chemotherapy.

Since it could be easily calculated from the routine clinical laboratory tests, testing the $\mathrm{CRP} / \mathrm{Alb}$ ratio could be used as a simple, feasible, inexpensive method to predict prognosis and to monitor the dynamic change of tumor burden. It might be possible that early management of systematic inflammation and nutritional support could increase the survival of patients with disseminated NPC. Since the nonsteroidal anti-inflammatory drugs (NSAIDs) have showed promising effect on preventing carcinogenesis and reducing the risk of death in various cancers [46-49], NSAIDs might be a feasible and convenient drug to modulate the inflammation of NPC patients. Moreover, whether dynamic change of the $\mathrm{CRP} / \mathrm{Alb}$ ratio during the treatment course could predict prognosis and treatment response remains unknown. Future prospective clinical trials and deeper basic research could 
address these questions and provide more reliable molecular and genetic mechanisms regarding the $\mathrm{CRP} / \mathrm{Alb}$ ratio.

There were several limitations to be acknowledged in this study. First, this was a retrospective study limited to a single institute. Second, another independent cohort was not introduced to validate the novel prognostic index. Finally, we could not provide complete information on disease-free interval (DFI), which was previously established as an important prognostic factor and thus did not further discuss it in our study. All the inferiorities should be overcome by prospective, multicentre studies.

\section{Conclusion}

In summary, our study demonstrated that the CRP/Alb ratio was an independent prognostic factor in metastatic NPC. The CRP and Alb levels could be easily, feasibly, inexpensively examined and therefore it is convenient to use the $\mathrm{CRP} / \mathrm{Alb}$ ratio in our clinical practice to evaluate systematic inflammation and predicate the survival outcome in metastatic NPC. In addition, we proposed a new prognostic score system based on both the GPS and the CRP/Alb ratio. Prospective multicentre studies are warranted to validate this model and to further explore the underlying mechanisms.

\section{Abbreviations}

ALP: $\quad$ Alkaline phosphatase

CRP/Alb: The ratio of CRP/albumin

CRP: $\quad$ C-reactive protein

DFI: Disease-free interval

EBV: $\quad$ Epstein-Barr virus

ESCC: Esophageal squamous cell carcinoma

GPS: $\quad$ Glasgow Prognostic Score

HRs: Hazard ratios

IMRT: Intensity modulated radiotherapy technology

KPS: $\quad$ Karnofsky Performance Scores

LDH: Lactate dehydrogenase

mGPS: $\quad$ Modified Glasgow Prognostic Score

MLR: $\quad$ Monocyte to lymphocyte ratio

NLR: $\quad$ Neutropil to lymphocyte ratio

NPC: $\quad$ Nasopharyngeal carcinoma

NSAIDs: Nonsteroidal anti-inflammatory drugs

PF: $\quad$ Cisplatin plus 5-fluorouracil

PLR: $\quad$ Platelet-to-lymphocyte ratio

PS: $\quad$ Performance status

RECIST: Response Evaluation Criteria in Solid Tumors

TNM: Tumor-node-metastasis

TP: $\quad$ Paclitaxel plus cisplatin

TPF: $\quad$ Paclitaxel plus cisplatin plus 5-fluorouracil.

\section{Conflicts of Interest}

The authors declare that they have no competing interests.

\section{Authors' Contributions}

Peng Sun and Cui Chen collected clinical data and drafted the manuscript. Yi Xia, Xiwen Bi, and Fei Zhang participated in the design of the study. Hang Yang, Panpan Liu, and Xin An performed the statistical analysis. Fenghua Wang and Wenqi Jiang conceived the study and participated in its design and coordination and helped to draft the manuscript. All authors read and approved the final manuscript. Peng Sun, Cui Chen, and Yi Xia contributed equally to this work.

\section{Acknowledgments}

The authors feel deep gratitude to all the staff members in their department for their support and suggestion in this study.

\section{References}

[1] W. Chen, R. Zheng, P. D. Baade et al., "Cancer statistics in China, 2015," CA: A Cancer Journal for Clinicians, vol. 66, no. 2, pp. 115-132, 2016.

[2] E. T. Chang and H. O. Adami, "The enigmatic epidemiology of nasopharyngeal carcinoma," Cancer Epidemiology, Biomarkers \& Prevention, vol. 15, no. 10, pp. 1765-1777, 2006.

[3] M. C. Yu and J. M. Yuan, "Epidemiology of nasopharyngeal carcinoma," Seminars in Cancer Biology, vol. 12, no. 6, pp. 421-429, 2002.

[4] P. Sun, C. Chen, Y. K. Cheng et al., "Serologic biomarkers of Epstein-Barr virus correlate with TNM classification according to the seventh edition of the UICC/AJCC staging system for nasopharyngeal carcinoma," European Archives of Oto-Rhino-Laryngology, vol. 271, no. 9, pp. 2545-2554, 2014.

[5] Y. Sun, L. L. Tang, L. Chen et al., "Promising treatment outcomes of intensity-modulated radiation therapy for nasopharyngeal carcinoma patients with N0 disease according to the seventh edition of the AJCC staging system," BMC Cancer, vol. 12, no. 1, p. 68, 2012.

[6] L. Zhang, Q. Y. Chen, H. Liu, L. Q. Tang, and H. Q. Mai, "Emerging treatment options for nasopharyngeal carcinoma," Drug Design, Development and Therapy, vol. 7, no. 4, pp. 3752, 2013.

[7] S. Rottey, I. Madani, P. Deron, and S. Van Belle, "Modern treatment for nasopharyngeal carcinoma: current status and prospects," Current Opinion in Oncology, vol. 23, no. 3, pp. 254-258, 2011.

[8] Y. Bensouda, W. Kaikani, N. Ahbeddou et al., "Treatment for metastatic nasopharyngeal carcinoma," European Annals of Otorhinolaryngology, Head and Neck Diseases, vol. 128, no. 2, pp. 79-85, 2011.

[9] J. Ma, Z. S. Wen, P. Lin, X. Wang, and F. Y. Xie, “The results and prognosis of different treatment modalities for solitary metastatic lung tumor from nasopharyngeal carcinoma: a retrospective study of 105 cases," Chinese Journal of Cancer, vol. 29, no. 9, pp. 787-795, 2010.

[10] Y. Jin, Y. X. Shi, X. Y. Cai et al., "Comparison of five cisplatinbased regimens frequently used as the first-line protocols in metastatic nasopharyngeal carcinoma," Journal of Cancer Research and Clinical Oncology, vol. 138, no. 10, pp. 17171725, 2012.

[11] C. Chen, F. H. Wang, X. An et al., "Triplet combination with paclitaxel, cisplatin and 5-FU is effective in metastatic and/or recurrent nasopharyngeal carcinoma," Cancer Chemotherapy and Pharmacology, vol. 71, no. 2, pp. 371-378, 2013. 
[12] C. Chen, F. H. Wang, Z. Q. Wang et al., "Salvage gemcitabine-vinorelbine chemotherapy in patients with metastatic nasopharyngeal carcinoma pretreated with platinumbased chemotherapy," Oral Oncology, vol. 48, no. 11, pp. 1146-1151, 2012.

[13] H. Zhang, W. Xia, X. Lu et al., “A novel statistical prognostic score model that includes serum CXCL5 levels and clinical classification predicts risk of disease progression and survival of nasopharyngeal carcinoma patients," PLoS One, vol. 8, no. 2, article e57830, 2013.

[14] S. H. Cheng, S. Y. Tsai, C. F. Horng et al., "A prognostic scoring system for locoregional control in nasopharyngeal carcinoma following conformal radiotherapy," International Journal of Radiation Oncology, Biology, Physics, vol. 66, no. 4, pp. 992-1003, 2006.

[15] C. C. Lee, T. T. Huang, M. S. Lee et al., "Clinical application of tumor volume in advanced nasopharyngeal carcinoma to predict outcome," Radiation Oncology, vol. 5, no. 1, p. 20, 2010.

[16] Y. K. Ong, D. M. Heng, B. Chung et al., "Design of a prognostic index score for metastatic nasopharyngeal carcinoma," European Journal of Cancer, vol. 39, no. 11, pp. 1535-1541, 2003.

[17] H. L. Ruan, H. D. Qin, Y. Y. Shugart et al., "Developing genetic epidemiological models to predict risk for nasopharyngeal carcinoma in high-risk population of China," PLoS One, vol. 8, no. 2, article e56128, 2013.

[18] L. Chen, L. Z. Liu, M. Chen et al., "Prognostic value of subclassification using MRI in the $\mathrm{t} 4$ classification nasopharyngeal carcinoma intensity-modulated radiotherapy treatment," International Journal of Radiation Oncology, Biology, Physics, vol. 84, no. 1, pp. 196-202, 2012.

[19] J. X. Li, S. M. Huang, B. X. Wen, and T. X. Lu, "Prognostic factors on overall survival of newly diagnosed metastatic nasopharyngeal carcinoma," Asian Pacific Journal of Cancer Prevention, vol. 15, no. 7, pp. 3169-3173, 2014.

[20] C. K. Toh, D. Heng, Y. K. Ong, S. S. Leong, J. Wee, and E. H. Tan, "Validation of a new prognostic index score for disseminated nasopharyngeal carcinoma," British Journal of Cancer, vol. 92, no. 8, pp. 1382-1387, 2005.

[21] Y. Jin, X. Y. Cai, Y. C. Cai et al., "To build a prognostic score model containing indispensible tumour markers for metastatic nasopharyngeal carcinoma in an epidemic area," European Journal of Cancer, vol. 48, no. 6, pp. 882-888, 2012.

[22] Y. Jin, X. Ye, L. Shao et al., "Serum lactic dehydrogenase strongly predicts survival in metastatic nasopharyngeal carcinoma treated with palliative chemotherapy," European Journal of Cancer, vol. 49, no. 7, pp. 1619-1626, 2013.

[23] W. Y. Wang, C. W. Twu, H. H. Chen et al., "Plasma EBV DNA clearance rate as a novel prognostic marker for metastatic/ recurrent nasopharyngeal carcinoma," Clinical Cancer Research, vol. 16, no. 3, pp. 1016-1024, 2010.

[24] C. L. Hsu, K. P. Chang, C. Y. Lin et al., "Plasma Epstein-Barr virus DNA concentration and clearance rate as novel prognostic factors for metastatic nasopharyngeal carcinoma," Head \& Neck, vol. 34, no. 8, pp. 1064-1070, 2012.

[25] W. X. Xia, Y. F. Ye, X. Lu et al., "The impact of baseline serum C-reactive protein and C-reactive protein kinetics on the prognosis of metastatic nasopharyngeal carcinoma patients treated with palliative chemotherapy," PLoS One, vol. 8, no. 10, article e76958, 2013.
[26] C. Chen, P. Sun, Q. S. Dai, H. W. Weng, H. P. Li, and S. Ye, "The Glasgow Prognostic Score predicts poor survival in cisplatin-based treated patients with metastatic nasopharyngeal carcinoma," PLoS One, vol. 9, no. 11, article e112581, 2014.

[27] T. Zhou, S. Hong, Z. Hu et al., "A systemic inflammationbased prognostic scores (mGPS) predicts overall survival of patients with small-cell lung cancer," Tumour Biology, vol. 36, no. 1, pp. 337-343, 2015.

[28] G. N. Lin, J. W. Peng, D. Y. Liu, J. J. Xiao, Y. Q. Chen, and X. Q. Chen, "Increased lymphocyte to monocyte ratio is associated with better prognosis in patients with newly diagnosed metastatic nasopharyngeal carcinoma receiving chemotherapy," Tumour Biology, vol. 35, no. 11, pp. 10849-10854, 2014.

[29] R. Jiang, X. Y. Cai, Z. H. Yang et al., "Elevated peripheral blood lymphocyte-to-monocyte ratio predicts a favorable prognosis in the patients with metastatic nasopharyngeal carcinoma," Chinese Journal of Cancer, vol. 34, no. 6, pp. 237-246, 2015.

[30] R. Malhotra, D. Marcelli, G. von Gersdorff et al., "Relationship of neutrophil-to-lymphocyte ratio and serum albumin levels with C-reactive protein in hemodialysis patients: results from 2 international cohort studies," Nephron, vol. 130, no. 4, pp. 263-270, 2015.

[31] M. H. Kim, J. Y. Ahn, J. E. Song et al., "The C-reactive protein/ albumin ratio as an independent predictor of mortality in patients with severe sepsis or septic shock treated with early goal-directed therapy," PLoS One, vol. 10, no. 7, article e0132109, 2015.

[32] X. Liu, X. Sun, J. Liu et al., "Preoperative C-reactive protein/ albumin ratio predicts prognosis of patients after curative resection for gastric cancer," Translational Oncology, vol. 8, no. 4, pp. 339-345, 2015.

[33] X. L. Xu, H. Q. Yu, W. Hu, Q. Song, and W. M. Mao, “A novel inflammation-based prognostic score, the C-reactive protein/ albumin ratio predicts the prognosis of patients with operable esophageal squamous cell carcinoma," PLoS One, vol. 10, no. 9, artcle e0138657, 2015.

[34] M. Ishizuka, H. Nagata, K. Takagi, Y. Iwasaki, N. Shibuya, and K. Kubota, "Clinical significance of the C-reactive protein to albumin ratio for survival after surgery for colorectal cancer," Annals of Surgical Oncology, vol. 23, no. 3, pp. 900-907, 2016.

[35] T. Zhou, J. Zhan, S. Hong et al., "Ratio of C-reactive protein/ albumin is an inflammatory prognostic score for predicting overall survival of patients with small-cell lung cancer," Scientific Reports, vol. 5, p. 10481, 2015.

[36] S. He, Y. Wang, H. Chen et al., "C-reactive protein/albumin ratio (CAR) as a prognostic factor in patients with nonmetastatic nasopharyngeal carcinoma," Journal of Cancer, vol. 7, no. 15, pp. 2360-2366, 2016.

[37] J. Budczies, F. Klauschen, B. V. Sinn et al., "Cutoff Finder: a comprehensive and straightforward web application enabling rapid biomarker cutoff optimization," PLoS One, vol. 7, no. 12 , article e51862, 2012.

[38] F. Colotta, P. Allavena, A. Sica, C. Garlanda, and A. Mantovani, "Cancer-related inflammation, the seventh hallmark of cancer: links to genetic instability," Carcinogenesis, vol. 30, no. 7, pp. 1073-1081, 2009.

[39] W. X. Xia, H. B. Zhang, J. L. Shi et al., "A prognostic model predicts the risk of distant metastasis and death for patients with nasopharyngeal carcinoma based on pre-treatment serum C-reactive protein and N-classification," European Journal of Cancer, vol. 49, no. 9, pp. 2152-2160, 2013. 
[40] T. Grimm, A. Buchner, B. Schneevoigt et al., "Impact of preoperative hemoglobin and CRP levels on cancer-specific survival in patients undergoing radical cystectomy for transitional cell carcinoma of the bladder: results of a single-center study," World Journal of Urology, vol. 34, no. 5, pp. 703-708, 2015.

[41] M. Haas, R. P. Laubender, P. Stieber et al., "Prognostic relevance of CA 19-9, CEA, CRP, and LDH kinetics in patients treated with palliative second-line therapy for advanced pancreatic cancer," Tumour Biology, vol. 31, no. 4, pp. 351-357, 2010.

[42] M. I. Koukourakis, G. Kambouromiti, D. Pitsiava, P. Tsousou, M. Tsiarkatsi, and G. Kartalis, "Serum C-reactive protein (CRP) levels in cancer patients are linked with tumor burden and are reduced by anti-hypertensive medication," Inflammation, vol. 32, no. 3, pp. 169-175, 2009.

[43] V. Asher, J. Lee, and A. Bali, "Preoperative serum albumin is an independent prognostic predictor of survival in ovarian cancer," Medical Oncology, vol. 29, no. 3, pp. 2005-2009, 2012.

[44] Z. Chen, Y. Shao, M. Fan et al., "Prognostic significance of preoperative C-reactive protein: albumin ratio in patients with clear cell renal cell carcinoma," International Journal of Clinical and Experimental Pathology, vol. 8, no. 11, pp. 14893-14900, 2015.

[45] X. L. Wei, F. H. Wang, D. S. Zhang et al., "A novel inflammation-based prognostic score in esophageal squamous cell carcinoma: the C-reactive protein/albumin ratio," $B M C$ Cancer, vol. 15, p. 350, 2015.

[46] H. Nan, C. M. Hutter, Y. Lin et al., "Association of aspirin and NSAID use with risk of colorectal cancer according to genetic variants," Jama, vol. 313, no. 11, pp. 1133-1142, 2015.

[47] R. C. Wender, "Aspirin and NSAID chemoprevention, geneenvironment interactions, and risk of colorectal cancer," Jama, vol. 313, no. 11, pp. 1111-1112, 2015.

[48] C. C. Johnson, M. Jankowski, S. Rolnick, M. U. Yood, and S. H. Alford, "Influence of NSAID use among colorectal cancer survivors on cancer outcomes," American Journal of Clinical Oncology, 2014.

[49] T. M. Brasky, M. R. Bonner, K. B. Moysich et al., "Non-steroidal anti-inflammatory drug (NSAID) use and breast cancer risk in the Western New York Exposures and Breast Cancer (WEB) study," Cancer Causes \& Control, vol. 21, no. 9, pp. 15031512, 2010. 


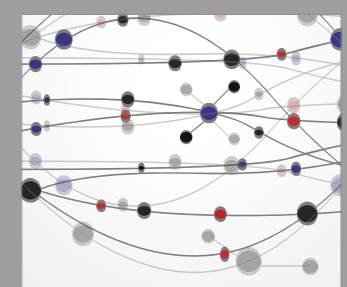

The Scientific World Journal
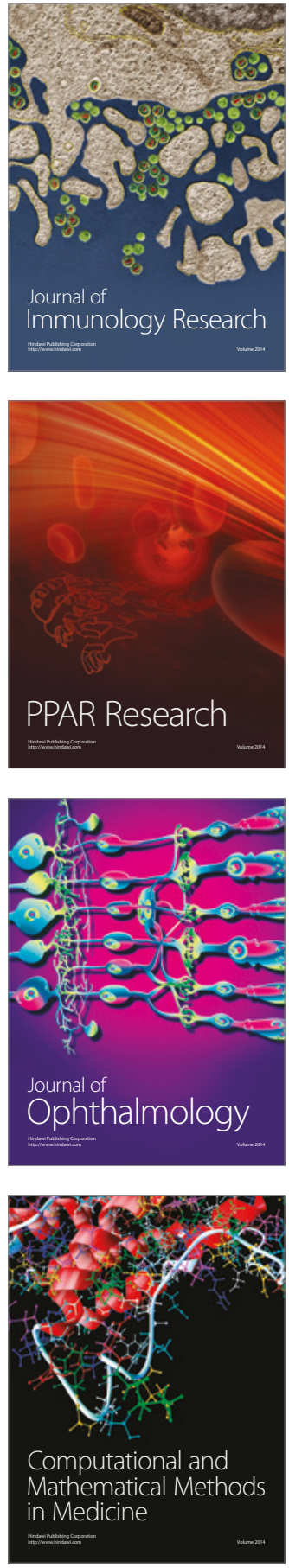

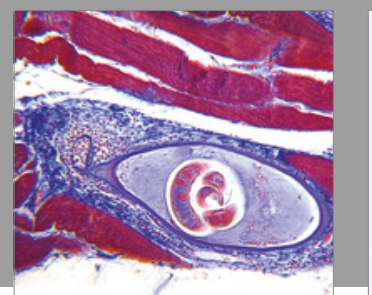

Gastroenterology Research and Practice
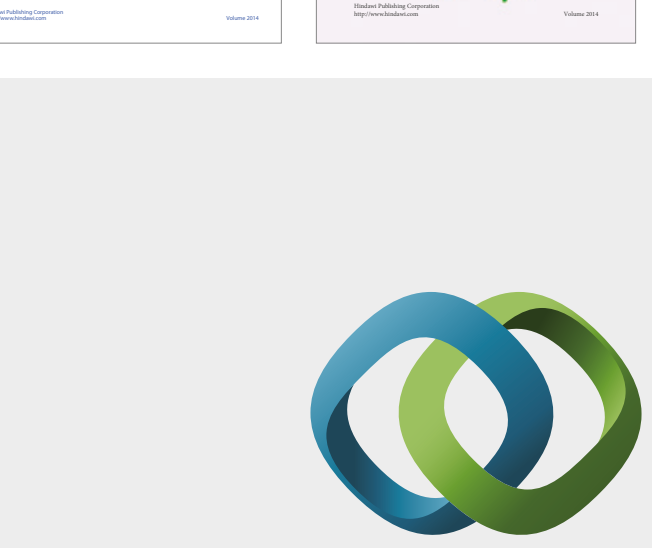

\section{Hindawi}

Submit your manuscripts at

https://www.hindawi.com
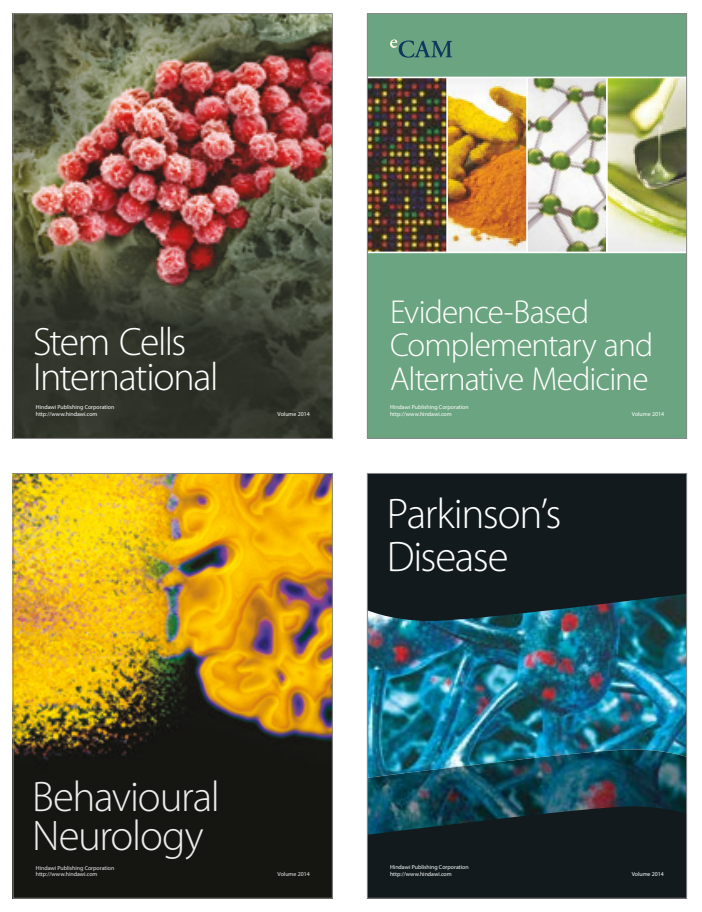
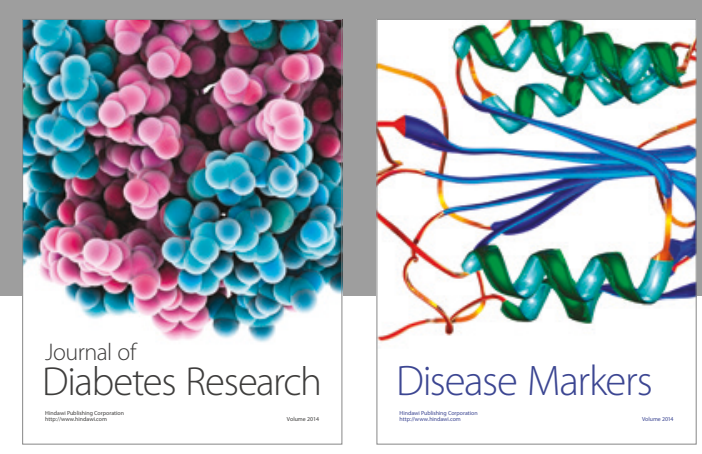

Disease Markers
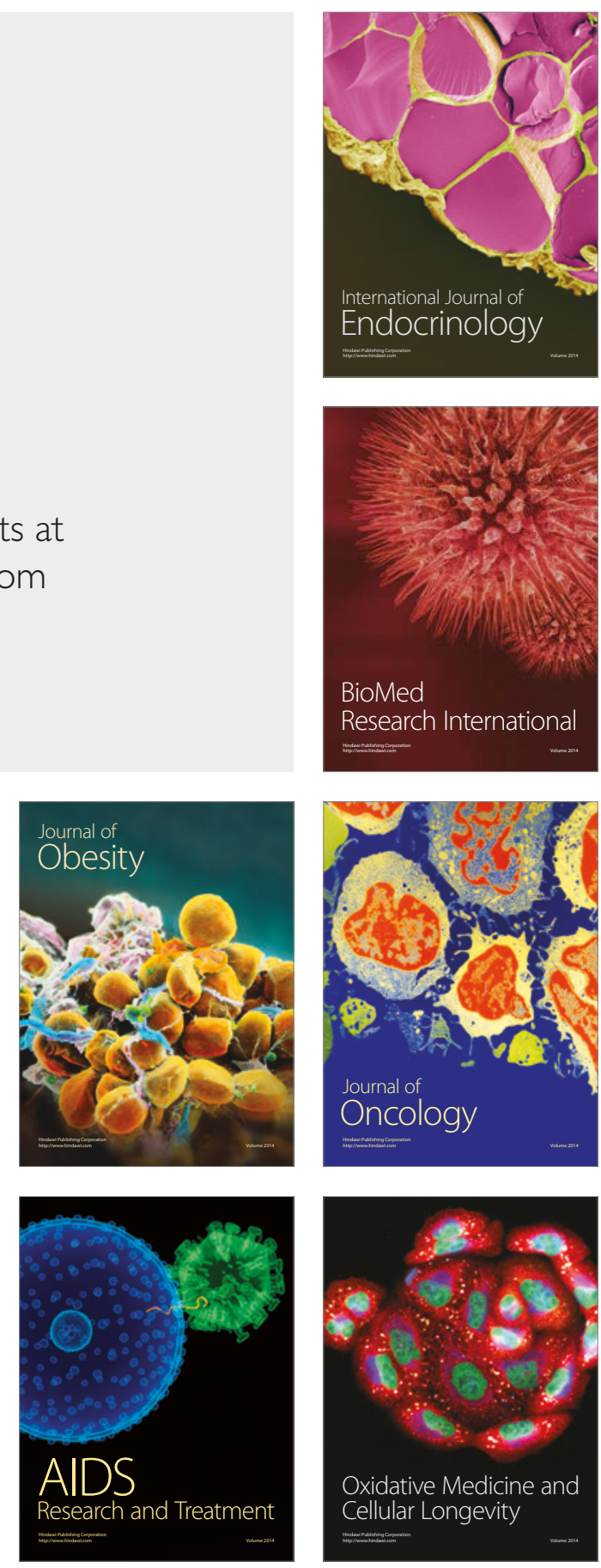